\title{
Validez y confiabilidad del cuestionario de ansiedad y miedo dental IDAF -4C+ en adultos mayores.
}

\section{Validity and reliability of the Index of Dental Anxiety and Fear (IDAF-4C+) in Chilean older adults.}

\author{
Matías Ríos-Erazo ${ }^{1}$, Andrea Herrera-Ronda ${ }^{1 *}$, Camila Farías ${ }^{2}$, Valeska Valdés ${ }^{3}$, Jacqueline Aguilar ${ }^{1}$, \\ Paula van Treek', Gonzalo Rojas-Alcayaga ${ }^{1}$
}

1. Área de Ciencias del Comportamiento, Instituto de Investigación en Ciencias Odontológicas, Facultad de Odontología, Universidad de Chile, Santiago, Chile.

2. Estudiante de Pregrado Carrera de Odontología, Facultad de Odontología, Universidad de Chile, Santiago, Chile.

3. Centro de Salud Familiar: Santa Amalia, Corporación Municipal de La Florida, La Florida, Chile.

* Correspondencia Autor: Andrea Herrera Ronda. | Dirección: Olivos 943, Independencia, Santiago, Chile. | Teléfono: +562 29781844 |

E-mail: aherrera@odontologia.uchile.cl.

Trabajo recibido el 22/06/2020.

Trabajo revisado 05/08/2020

Aprobado para su publicación el 21/08/2020
En el artículo titulado "Validez y confiabilidad del cuestionario de ansiedad y miedo dental IDAF-4C+ en adultos mayores" de los autores Matías Ríos-Erazo, Andrea Herrera-Ronda, Camila Farías, Valeska Valdés, Jacqueline Aguilar, Paula van Treek y Gonzalo Rojas-Alcayaga, publicado en el vol. 14, №1 de Abril 2021, se menciona a lo largo del artículo el análisis exploratorio confirmatorio y debiera decir análisis exploratorio.

DOI: $10.4067 / S 2452-55882021000100022$ 